\title{
M13 Virus Based SERS Nanoprobe for Multiplexed and Quantitative Immunoassay
}

\author{
Hye-Eun Lee ${ }^{1}$, Norov Erdene ${ }^{2}$, Hyori Kim³ ${ }^{3}$ Yoon-Sik Lee ${ }^{4}$, Junho Chung ${ }^{3}$, \\ Dae Hong Jeong ${ }^{2}$, Ki Tae Nam ${ }^{1}$ \\ ${ }^{1}$ Department of Materials Science and Engineering, Seoul National University, Seoul 151-747, Korea; ${ }^{2}$ Department of Chemistry \\ Education, Seoul National University, Seoul 151-747, Korea; ${ }^{3}$ Department of Biochemistry and Molecular Biology and School of \\ Medicine \& Cancer Research Institute, Seoul National University, Seoul 110799, Korea; ${ }^{4}$ School of Chemical and Biological \\ Engineering, Seoul National University, Seoul 151-747, Korea; (nkitae@snu.ac.kr)
}

Surface-enhanced Raman scattering (SERS)-based immunoassay offers unprecedented opportunities for multiplexing immunoassays because Raman spectrum exhibit sharp peak width compared to fluorescence. However, there are still big challenges in the development of well-defined SERS probes that exhibit the reproducible and quantifiable signals.

Typically, preparation of SERS probe involves several steps such as the functionalization of gold nanoparticle, immobilization of antibodies, and labeling with Raman reporter molecules. During this several conjugation procedures there is no method to control precise number and orientation of conjugating moieties. Consequently randomly attached antibody on SERS probe leads to irregular binding affinity for each probe and uncertainty in SERS signals.

Herein, we show a new approach, based on M13 virus, to manufacture SERS probe which has the same quality for all probes, higher signal intensity, and most importantly controlled orientation of the antibodies for the quantitative assay.

A key advantage of using M13 virus lies within their intrinsic structure which can be easily modified chemically and genetically. By utilizing different part of M13 virus coat proteins for different purpose, M13 virus-based SERS probe provides significant advantages: (i) high signal enhancement with uniformity of Raman signal and (ii) controlled orientation and number of the antibodies on SERS probe. On coat protein VIII part, virus has multiple copies of binding group in a well-defined geometry. This allows the way to array gold nanoparticles in a highly ordered fashion with close junction where strong electromagnetic enhancement occurs. Unlike the conventional SERS probes, the presented probe can have exact number and orientation of antibody on its coat protein III part. Therefore, not only detecting antigen can be maximized by these fully accessible antibodies but also quantitative analysis can be possible. 